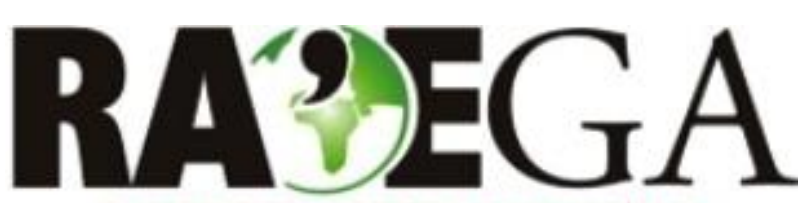

O ESPACO GEOGRÁFICO EM ANÁL.ISE

\title{
A IMPORTÂNCIA DOS PARQUES URBANOS E ÁREAS VERDES NA PROMOÇÃO DA QUALIDADE DE VIDA EM CIDADES
}

\section{THE IMPORTANCE OF URBAN PARKS AND GREEN AREAS IN IMPROVING THE QUALITY OF LIFE IN CITIES}

\author{
Bani Szeremeta \\ Professor Doutor da Área de Ciências Ambientais \\ Serviço Nacional de Aprendizagem Comercial - SENAC \\ Curitiba, PR, Brasil \\ e-mail: bani@ufpr.br
}

Artigo recebido em: 02/02/2013.

\author{
Paulo Henrique Trombetta Zannin \\ Professor Doutor da Área de Acústica - Coordenador do Laboratório \\ de Acústica Ambiental - Industrial e Conforto Acústico - UFPR \\ Curitiba, PR, Brasil \\ e-mail:paulo.zannin@gmail.com
}

Artigo aceito em: 28/10/2013.

\section{Resumo}

Os parques urbanos são áreas verdes que podem trazer qualidade de vida para a população. Pois proporcionam contato com a natureza e suas estruturas e qualidade ambiental, quando adequadas e atrativas, são determinantes para a realização de atividade física e o lazer. Estas atividades trazem diferentes benefícios psicológicos, sociais e físicos a saúde dos indivíduos, como, por exemplo, a redução do sedentarismo e amenizar o estresse do cotidiano urbano. Assim, o planejamento correto e a conservação de parques públicos se revelam como significativa estratégia para uma política efetiva do projeto urbano e da saúde pública. O objetivo deste artigo, por meio de uma revisão não sistemática, foi identificar os fatores sociais e ambientais dos parques que os tornam mais atrativos para a atividade física. Constatou-se que a beleza da paisagem e a proximidade de um parque, ao local de moradia dos usuários, são os principais fatores que incentivam uma utilização frequente para a atividade física e o lazer.

Palavras-chave: Ambiente urbano, parque urbano, saúde, atividade física. 


\begin{abstract}
The urban parks are green areas that can bring quality of life for the population. They provide contact with nature and its structures and environmental quality, and attractive when appropriate, are crucial for physical activity and recreation. These activities bring different benefits psychological, social and physical health of individuals, such as reducing sedentary lifestyle and alleviate the stress of everyday urban life. Thus, the correct planning and conservation of public parks are revealed as significant strategy for an effective policy of urban design and public health. This paper, through a non-systematic review was to identify the social and environmental factors that make the parks more attractive for physical activity. It was found that the beauty of the landscape and the proximity of a green area, housing location of users, are the main factors that encourage repeated use for physical activity and recreation.
\end{abstract}

Keywords: Urban environment, urban park, health, physical activity.

\title{
INTRODUÇÃO
}

Os parques que apresentam condições ambientais adequadas são determinantes na utilização de parques para o desenvolvimento de atividades físicas e o lazer. Ou seja, podem contribuir na redução da prevalência de sedentarismo e auxiliar na promoção da saúde e bem estar, além de possibilitar o aumento do nível de atividade física dos ativos. Em contrapartida, a má qualidade do ambiente e a insatisfação dos usuários são determinantes ambientais negativos para o uso dos parques, de forma a vir descaracterizar estas funções associadas à qualidade de vida e saúde pública.

Neste contexto, alguns estudos mostram que a boa qualidade social e física destes espaços, como por exemplo, infra-estrutura adequada, segurança, facilidade de acesso e outros fatores positivos, aumentam a possibilidade de freqüência das pessoas e, por conseguinte, um comportamento fisicamente ativo (REIS, 2001; FISHER et al., 2004; BEDIMO-RUNG et al., 2005; HORNIG, 2005; SALLIS et al., 2006; BAKER et al., 2008; COLLET et al., 2008; CASSOU, 2009; TESTER et al., 2009; COHEN et al., 2010).

Os parques urbanos, por suas características físicas e sociais, são considerados apropriados para a prática de atividade física ao ar livre e recreação. Segundo Barton e Pretty (2010), apenas cinco minutos de caminhada em áreas verdes, como por exemplo, em um parque público, já é suficiente para melhorar a 
saúde mental, com benefícios para o humor e autoestima. Ainda, outros estudos apresentam diferentes benefícios (sociais, físicos e psicológicos) de utilizar espaços naturais ou ambientes urbanos com áreas verdes para a prática destas atividades, como por exemplo: educação ambiental, reduzir a prevalência de sedentarismo e amenizar o estresse (KAPLAN, 1995; BODIN; HARTIG, 2003; STAATS et al., 2003; HERZOG et al., 2003; PRETTY et al., 2005; BELL et al., 2005; BEDIMO-RUNG et al., 2005; COHEN et al., 2007; HANSMANN et al., 2007).

Estas áreas urbanas podem ser consideradas "academias ao ar livre". Assim, a implantação das mesmas é de relevante importância na promoção da saúde e qualidade de vida de uma população. No entanto, percebe-se que além de políticas públicas que incentivem a construção e revitalização destes espaços, são de igual importância projetos que contemplem planejamentos e gestões que supram as necessidades dos seus frequentadores e comunidade em geral. Ou seja, é preciso que estes ambientes sejam percebidos positivamente para que as pessoas se sintam atraídas e motivadas a frequentá-los, e também desfrutem, de forma satisfatória, dos benefícios que o desenvolvimento de atividades nestes locais pode proporcionar (REIS, 2001; COHEN et al., 2007; CASSOU, 2009).

Assim, esta revisão visa destacar a importância de áreas verdes de lazer na promoção da atividade física nas comunidades, de modo a justificar a relevância de pesquisas sobre a qualidade ambiental de parques urbanos que são comumente utilizados para a realização de atividades ao ar livre. Considerando-se que a atividade física traz vários benefícios à saúde e qualidade de vida (WARBURTON et al., 2006), e a implantação, e os corretos planejamentos e conservação de parques públicos se revelam como significativa estratégia para uma política efetiva do projeto urbano e da saúde pública.

\section{MÉTODOS}

Para identificar e selecionar os estudos considerados relevantes ao desenvolvimento desta pesquisa foram realizadas buscas, a partir do segundo semestre de 2010, nas bases de dados eletrônicas Medline/Pubmed, Scielo e LILACs, utilizando os seguintes descritores (na língua inglesa e portuguesa): 
atividade física, ambiente, áreas verdes, parques públicos, parques urbanos, espaços públicos, qualidade de vida, saúde.

Também foram diretamente acessados e consultados alguns sites de periódicos da área de educação física, da saúde e meio ambiente, assim como buscados, manualmente, materiais impressos e digitais (CDs) nas bibliotecas da Universidade Federal do Paraná (UFPR).

Deste modo, foram pesquisadas diferentes fontes (artigos, teses, dissertações e livros), de modo a satisfazer os princípios básicos necessários para a realização da pesquisa. As fontes primárias foram identificadas com base no seu título, sendo selecionados todos os resumos considerados previamente relevantes e somente analisados os que apresentaram data de publicação (artigos) ou defesa (tese) até 30/06/2010 (sem data inicial). Após essa etapa, todos os artigos selecionados foram obtidos na íntegra e examinados. Por último, realizaram-se pesquisas pelo nome do primeiro autor desses manuscritos para identificar outras fontes que estivessem dentro dos critérios de inclusão. Outra alternativa foi consultar as referências bibliográficas de cada artigo escolhido, a fim de encontrar alguma fonte de importância para o estudo.

\section{RESULTADOS E DISCUSSÃO}

As áreas verdes, em relação as suas características, podem interferir no cidadão e no ambiente urbano de muitas formas, mostrando-se de grande importância para a comunidade, já que este tipo de espaço, atualmente, remete à melhor qualidade de vida (CROMPTON, 2001; HARNIK, 2003; SHERER, 2003; BEDIMO-RUNG et al., 2005; LIBRETT et al., 2007). Os parques são caracterizados como um tipo de área verde urbana, pois apresentam predomínio de vegetação (independente do porte) que integram o ambiente construído, além de possuírem outras características naturais. Possuem na cidade diferentes funções, sendo as principais: ecológica, estética e lazer (NUCCI, 2001; MASCARÓ, 2002).

Conforme Milano (1984), a vegetação é responsável pela criação de ambientes esteticamente agradáveis, valorizando uma área e atuando como elemento que ameniza o estresse. O urbanismo contemporâneo gera a necessidade da existência de espaços verdes para que exista a possibilidade de fugir do ruído e 
da poluição, de forma a regressar à natureza (CUNHA, 1997). De modo similar, Andrade (2001) afirma que estes locais são uma forma de refúgio, a valorização do ambiente natural em meio do ambiente construído.

Assim sendo, as áreas verdes tornam-se referências nos grandes centros urbanos, estando mais associadas à função recreativa, porque oferecem diversos tipos de atividades - como, por exemplo, caminhadas, jogos e relaxamento -, além de funcionarem como ponto de socialização (ANDRADE, 2001; CASSOU, 2009). Portanto, considerando-se a necessidade de espaços mais adequados para a prática de atividade física ao ar livre, bem como para um lazer satisfatório, os parques urbanos, as praças públicas e outras áreas naturais (praias) são os locais que apresentam os maiores potenciais para estes objetivos (SOUZA, 2007).

Neste contexto, cada vez mais pesquisas associam os espaços verdes a promoção da atividade física. Por exemplo, Corti et al. (1997), mencionam que os parques provavelmente estimulariam a atividade física por fatores relacionados à motivação, uma vez que as pessoas estariam mais satisfeitas com os caminhos compostos de vegetação arbórea do que com os espaços vazios. Também há evidências de que o acesso a estas áreas colabora para aumentar os níveis de atividade física (GILES-CORTI et al., 2005; DESHPANDE et al., 2005; COHEN et al., 2007; SOARES, 2009; MCCORMACK et al., 2010).

Ainda, outros estudos apresentam as diferentes vantagens para o bem estar e a saúde mental, resultantes da prática de atividade física em espaços naturais ou ambientes urbanos constituídos de áreas verdes (KAPLAN, 1995; HERZOG et al., 2002; BODIN; HARTIG, 2003; STAATS et al., 2003; PRETTY et al., 2005; BELL et al., 2005; BEDIMO-RUNG et al., 2005; COHEN et al., 2007; HANSMANN et al., 2007; BARTON; PRETTY, 2010).

Barton e Pretty (2010) determinaram, por meio de um estudo de meta-análise, que apenas a "dose" de cinco minutos de exercício em áreas naturais ("exercício verde") é suficiente para trazer melhorias em indicadores da saúde mental (humor e autoestima), sugerindo benefícios imediatos. Ademais, constataram que a atividade física próxima de ecossistemas aquáticos fornece melhores efeitos, apesar de que todos os tipos de áreas com presença de natureza apresentaram resultados satisfatórios. Tais evidências sugerem que as pessoas, hoje sedentárias e/ou com 
problemas de natureza psíquica, teriam benefícios na saúde mental se comprometendo a exercícios de curta duração em espaços verdes acessíveis.

Os autores também enfatizam que urbanistas e arquitetos devem melhorar o acesso a estas áreas ("desenho verde") e que precisam ser oferecidas às crianças oportunidades de aprender em ambientes externos ("educação verde") (BARTON; PRETTY, 2010). Além disso, campanhas de saúde pública podem incentivar as pessoas a utilizarem os espaços verdes com maior assiduidade e praticar exercícios ao ar livre. Sendo que as melhorias à saúde, atribuída a estes ambientes, devem ser mais conhecidas na busca de os tornarem mais eficazes junto à população (HANSMANN et al., 2007).

Hansmann et al. (2007) analisaram, a partir de inquéritos, mudanças de curto prazo no bem estar subjetivo e na percepção dos níveis de estresse agudo relacionados a uma única experiência de visita em dois espaços verdes diferentes, um parque e uma floresta. Não constataram diferenças estatísticas nos efeitos de reparação entre os ambientes estudados. Mas, observaram que o estresse agudo de diferentes amplitudes pode ser efetivamente reduzido visitando estas áreas. 0 exercício de maior intensidade (vigoroso) foi associado com uma maior redução do estresse e aumento da sensação de bem estar ("equilíbrio mental") em comparação as demais atividades analisadas (caminhada, relaxar, observar a natureza).

Assim, percebe-se que os parques urbanos são considerados próprios para o desenvolvimento de atividades físicas, podendo ser considerados verdadeiras "academias ao ar livre". Desde apenas uma visita até a prática regular de exercícios físicos em áreas verdes ("exercício verde") é possível verificar importantes benefícios a saúde física e mental. O que torna imprescindível a implantação destes espaços para ajudar na melhoraria da saúde coletiva e bem estar.

Porém, de acordo com Nahas (2003), atualmente a aglomeração urbana, o desenvolvimento da tecnologia e a consequente redução de espaços livres estabeleceram ambientes que tendem a direcionar os indivíduos a um estilo de vida inativo. Segundo Collet et al. (2008), nos dias de hoje é um desafio à manutenção de ambientes saudáveis que estimulem a prática regular de atividades físicas. Neste caso, percebe-se que os poucos lugares dos grandes centros urbanos que mantêm 
estas características ambientais devem ter especial cuidado de todos os segmentos da sociedade, responsáveis pela conservação destas áreas.

Em razão disto, compreende-se que apenas a criação de parques não garante a promoção do lazer e da atividade física. Para alcançar estes objetivos, devem ser realizados projetos e ações que apreciem as necessidades, opiniões e anseios dos usuários e da comunidade em geral (HILDEBRAND, 2001). Ou seja, na atualidade, não basta apenas a iniciativa própria dos cidadãos em fazer exercícios ao ar livre. A cidade deve oportunizar áreas, através de um planejamento urbano eficiente, com condições para que as pessoas os realizem de modo regular (KAPLAN et al., 1995; FRUMKIN, 2003; HANSMANN et al., 2007).

Em outras palavras, é necessário que os parques apresentem uma infraestrutura apropriada, programação de atividades, ambientes agradáveis e salubres, e facilidade de acesso (entre outros fatores positivos), para que com isto as pessoas se sintam atraídas e motivadas a frequentá-los (FISHER et al., 2004; PRETTY et al., 2005; DAWSON et al., 2007; KAMPHUIS et al., 2007, COHEN et al., 2007; CASSOU 2009, COHEN et al., 2010). Esta qualidade sócio-ecológica, que implica a relação complexa entre o homem e o meio, que ajudará a estabelecer uma importante estratégia de uma política de saúde pública eficaz, de modo a reduzir a prevalência de sedentários, a aumentar os níveis de atividade física e, por consequência, melhorar a qualidade de vida da população (STOKOLS, 1992; SALLIS et al., 2006). Haja vista, o sedentarismo e a falta de atividade física em quantidades necessárias são fatores que podem ser associados ao sobrepeso e desenvolvimento da obesidade (U.S. Department of Health and Human Services, 1996), bem como ao decorrente aumento do risco ou evolução de doenças crônicas não transmissíveis (BERLIN; COLDITZ, 1990; BLAIR et al., 1995; KOHL, 2001; BAUMAN, 2004; WARBURTON et al., 2006).

Diante disso, fica evidente que a condição ambiental é um importante indicador de qualidade de vida pelo fato de poder influenciar um comportamento fisicamente ativo (SALLIS et al., 1997; KAHN et al., 2002; OWEN et al., 2004; BEDIMO-RUNG et al., 2005; GODBEY et al., 2005; HOEHNER et al., 2005; GILESCORTI et al., 2005; SALLIS et al., 2006; KACZYNSKI; HENDERSON, 2007; MOWEN et al., 2008; CASSOU, 2009). As dinâmicas sociais, ambientais e culturais 
são interdependentes e influenciam no comportamento e atitudes do ser humano. De acordo com Soares (2009), a investigação da interdependência da atividade física, saúde e meio ambiente se resulta num elemento essencial na sociedade, permitindo, assim, recomendar medidas eficazes para a saúde coletiva, colaborando com o bem estar de todos.

Neste sentido, estudos, principalmente de origem internacional, focaram-se na pesquisa de fatores sócio-ambientais que podem ser determinantes no uso de parques e também na realização de atividades físicas regulares nestes espaços (KACZYNSKI; HENDERSON, 2007; MCCORMACK, et al. 2010). Ou seja, buscaram conhecer e entender as características e condições físicas e sociais do ambiente que podem influenciar o comportamento de um estilo de vida saudável, considerando-se a percepção e o perfil dos usuários.

Assim, atributos como a oferta de atividades programadas (CHOY et al., 2008; COHEN et al., 2010), facilidade de acesso e proximidade de casa (REIS, 2001; GILES-CORTI et al., 2005; COHEN et al., 2007; KACZYNSKI; HENDERSON, 2007; BAKER et al., 2008;) tamanho do parque (GILES-CORTI et al., 2005), potencial de ambiente não poluído (COLLET et al., 2008), disponibilidade de recursos e equipamentos (HORNIG, 2005; BEDIMO-RUNG et al., 2005; BAKER et al., 2008; KACZYNSKI; HENDERSON, 2008), existência de espaços verdes ou naturais (HORNIG, 2005), estética (KACZYNSKI; HENDERSON, 2008; COLLET et al., 2008), e manutenção (TESTER; BAKER, 2009) destacam-se como sendo capazes de promover o uso do parque e atividade física. Mas, características antagônicas a estas e outros fatores negativos podem impedir ou prejudicar o uso, como por exemplo: a poluição (REIS, 2001), presença de lixo, vandalismo e manutenção ineficiente (DAWSON et al., 2007).

Contudo, é importante ressaltar que as barreiras e facilitadores para a prática de atividade física, identificados em um determinado parque, são peculiares, pois o modo como são percebidos depende também de características demográficas, sociais e regionais de cada local (MCGINN et al., 2007; KIRCHHOFF et al., 2008). No Brasil, ainda são raras as publicações que dissertam sobre a importância da qualidade dos parques urbanos para a atividade física, sendo principalmente 
originadas por estudos realizados no sul do país (REIS, 2001; COLLET et al., 2008; CASSOU, 2009).

Reis (2001) analisou os determinantes sócio-ambientais para a realização de atividades físicas em usuários do Parque Jardim Botânico da cidade de Curitiba, capital do Paraná. Ainda, verificou os motivos de utilização e a associação desses fatores determinantes percebidos pelos sujeitos com variáveis sócio-demográficas e nível de atividade física. Constatou que a proximidade do parque da residência é o principal motivo de uso e os fatores que estimulam a prática de atividade física na área se apresentam em maior proporção e variedade em relação aos que inibem. A incidência de chuvas e a poluição do ar foram percebidas como barreiras (inibidores) para a atividade física. Já a localização do parque, beleza geográfica e das estruturas, pista de caminhada/corrida, estacionamentos, comportamento dos usuários, apoio/incentivo dos amigos e a regulamentação do trânsito nos arredores foram percebidos como fatores de estímulo, independente das pessoas praticarem ou não atividade física regularmente. O autor concluiu que os fatores ambientais presentes no parque parecem ser importantes para a realização de atividades físicas, mas sua percepção enquanto barreira ou estímulo - devido às associações encontradas no estudo - é dependente das condições econômicas, culturais e sociais e também dos sujeitos.

Outro estudo mais recente em Curitiba encontrou associação entre a frequência de usuários de parques e praças e a qualidade do ambiente (CASSOU, 2009). Sendo que na medida em que o potencial de qualidade do ambiente aumenta, maior é proporção de mulheres, pessoas mais velhas, escolarizadas e frequência de utilização. A respeito dos motivos para o uso, a maioria dos usuários de parques indicou ser a beleza e presença de estacionamento os fatores essenciais. Já os frequentadores de praças as utilizam principalmente pela proximidade de casa, estrutura, equipamentos e segurança.

No estado de Santa Catarina, Collet et al. (2008) analisaram, por meio do mesmo questionário de Reis (2001) - o qual contém uma "escala de percepção do ambiente existente", frequentadores de um parque na cidade de Florianópolis, considerando as quatro estações do ano. Destacando-se as qualidades físicas do ambiente, a disponibilidade de equipamentos e o estacionamento foram julgados 
pela maioria dos usuários como fatores que pouco estimulam a realização de atividades físicas no local em todas as estações. Por outro lado, os fatores físicos mais relevantes para o estímulo à prática de atividades físicas, independentemente do período do ano, foram: localização do parque; estética geográfica e arquitetônica; e o potencial de não haver poluição sonora, visual ou atmosférica. Além disso, similar aos resultados de Reis (2001), a conduta dos usuários, apoio dos amigos e familiares e a imagem atribuída ao parque foram fatores que incentivam os usuários a praticarem atividades físicas na área.

Szeremeta e Zannin (2009) avaliaram o ambiente sonoro de quatro parques de Curitiba-PR ladeados e/ou muito próximos de vias de intenso fluxo de veículos. Verificaram que todas estas áreas apresentavam médias de níveis de pressão sonora equivalente (entre 56 e $64 \mathrm{~dB}[\mathrm{~A}]$ ) superiores ao permitido pela legislação do município para áreas verdes, mesmo em período considerado mais tranquilo (entre 14 e 17 horas) que foram realizadas as medições acústicas. Entretanto, a maioria dos 335 usuários entrevistados nos quatro ambientes, julgou o "volume do som ambiente" como normal (71\%) e que não incomoda (81\%). Os parques tem se tornado um refúgio urbano, frente ao crescente ruído nas cidades (ZANNIN e SZEREMETA, 2003; da PAZ et al., 2005; GUEDES et al., 2011; ZANNIN e SANT'ANA, 2011; ZANNIN et al., 2013).

Por outro lado, quando as fontes sonoras foram avaliadas separadamente, 0 ruído de tráfego do entorno, julgado pela maioria dos inquiridos como desagradável $(84,2 \%)$, foi o segundo tipo de som mais identificado pelos mesmos $(28,5 \%$, precedido pelo canto de pássaros (32,6\%). Os autores também enfatizaram a importância de sempre associar dados objetivos (parâmetros acústicos) com dados subjetivos (percepção sonora) para o melhor entendimento da relação da paisagem sonora de parques urbanos com os visitantes, de modo a obter conhecimento para otimizar o projeto acústico e assim tornar o ambiente mais atrativo para o uso da comunidade.

Diante destas evidências sobre a importância dos parques e de suas qualidades para a promoção da atividade física e saúde, compreende-se que reconhecer e analisar estas qualidades ambientais, tal como as características e a percepção dos seus usuários e comunidade em geral (BEDIMO- RUNG et al., 2005), 
é a melhor estratégia para adequá-los em relação as suas funções e usos. Somente assim, a comunidade poderá usufruir de modo efetivo dos benefícios, até mesmo imediatos, que a utilização destes ambientes pode proporcionar.

\section{CONCLUSÃO}

Esta revisão não sistemática demonstrou que a utilização de parques urbanos possibilita melhoras na qualidade de vida da população. Observa-se que os benefícios sociais, físicos e psicológicos são satisfatórios para a comunidade que os utiliza. No entanto, o uso destas áreas depende de vários fatores sociais e ambientais presentes no ambiente destas áreas e características individuais dos seus usuários (idade, condições socioeconômicas, gênero, escolaridade, etc).

A maior proximidade as residências e a beleza da paisagem dos parques parecem ser os principais determinantes para a adesão e manutenção de frequentadores assíduos. Neste sentido, os parques devem ser implantados e planejados de acordo com o perfil e as necessidades da comunidade, além de serem estabelecidas políticas eficientes de conservação ambiental dessas áreas, já que a beleza da paisagem é atribuída pela presença de suas condições naturais (vegetação, lagos, relevo, etc), as quais podem também promover bem estar psicológico no visitante.

Estas ações devem aumentar a percepção positiva pela comunidade para que assim estes espaços públicos sejam efetivamente utilizados, possibilitando maiores níveis de atividade física e experiências psicológicas relevantes para a melhoria da saúde mental. Diante disso, verifica-se a maior necessidade da realização de pesquisas interdisciplinares com o envolvimento de especialistas de diferentes áreas de estudo (saúde, biológicas, engenharias, humanas).

\section{REFERÊNCIAS BIBLIOGRÁFICAS}

ANDRADE, R. V. O Processo de Produção dos Parques e Bosques Públicos de Curitiba. Curitiba, 2001. 120 p. Dissertação (Mestrado em Geografia) - Setor de Ciências da Terra, Universidade Federal do Paraná.

BAKER, E. A.; SCHOOTMAN, M.; KELLY, C.; and BARNIDGE, E. Do Recreational Resources Contribute to Physical Activity? Journal of Physical Activity and Health, vol.5, pag. 252-261, 2008. 
BARTON, J., PRETTY, J. What is the Best Dose of Natureand Green Exercise for Improving Mental Health? A Multi-Study Analysis. Environ. Sci. Technol, 44, 39473955, 2010.

BAUMAN, A.E. Updating the evidence that physical activity is good for health: an epidemiological review 2000-2003. Journal of Science and Medicine in Sport 7, 6-19, 2004.

BEDIMO-RUNG, A; MOWEN, A; COHEN, D. The significance of parks to physical activity and public health: a conceptual model. American Journal of Preventive Medicine. vol 28 (2 suppl 2) pag.159-168, 2005.

BELL, S., WARD THOMPSON, C., FINDLAY, C., MONTARZINO, A., MORRIS, N. Self reported stress reduction by users of woodlands. In: Gallis, C.T. (Ed.), Forests, Trees, and Human Health and Well-Being: 1st European COST E39 Conference Proceedings. Medical and Scientific Publishers, Thessaloniki, pp. 71-80, 2005.

BERLIN JA, COLDITZ GA. A Meta-Analysis of Physical Activity in the Prevention of Coronary Heart Disease. Am J Epidemiol, 132 (4): 612-628, 1990.

BLAIR SN, KOHL HW, BARLOW CE, et al. Changes in physical fitness and all-cause mortality: a prospective study of healthy and unhealthy men. JAMA, 273 (14): 10991105, 1995.

BODIN, M., HARTIG, T. Does the outdoor environment matter for psychological restoration gained through running? Psychology of Sport and Exercise 4, 141153, 2003.

CASSOU, A.C.N. Características ambientais, Frequência de utilização e nível de atividade física dos usuários de parques e praças de Curitiba-PR. Curitiba, 2009. 130 p. Dissertação (Mestrado em Educação Física) - Universidade Federal do Paraná.

CORTI, B., DONOVAN, R. J., HOLMAN, C. D. J. Factors influencing the use of physical activity facilities: Results from qualitative research. Health Promotion Journal of Australia, 7, 16-21, 1997.

CROMPTON, J.L. The impact of parks on property values: A review of the empirical evidence. Journal of Leisure Research, vol.33, n.1, pag.1-31, 2001.

COHEN, D. A.; MCKENZIE T.L.; SEHGAL, A., WILLIAMSON, S.; GOLINELLI, D. \& LURIE, N. Contribution of Parks to Physical Activity. American Journal of Public Health, vol. 97, pag. 509-514, 2007.

COHEN, D. A., MARSH, T., WILLIAMSON, S., DEROSE, K. P., MARTINEZ, H., SETODJI, C., MCKENZIE T.L. Parks and physical activity: Why are some parks used more than others? Preventive Medicine 50, S9-S12, 2010. 
COLLET, C.; CHIARADIA, B.M.; REIS, R.S.; NASCIMENTO, J.V.; Fatores Determinantes para a Realização de Atividades Físicas em Parque Urbano de Florianópolis. Revista Brasileira de Atividade Física \& Saúde, Vol. 13, N 1, 2008.

CHOY, L.B.; MCGURK, M.D.; TAMASHIRO, R.; NETT, B.; MADDOCK, ; J.E. Increasing Acess to Places for Physical Activity Through a Joint Use Agreement: A Case Study in Urban Honolulu. Preventing Chronic Disease, vol 5 suppl. 3, 2008. Disponível em: http://www.cdc.gov/pcd/issues/2008/jul/07_0117.htm. Acessado em: 14 de jul. 2010.

CUNHA, L. O espaço, o desporto e o desenvolvimento. Edições FMH, Lisboa, 1997.

da PAZ, EC; FERREIRA, AMC; ZANNIN, PHT. Estudo comparativo da percepção do ruído urbano. Revista de Saúde Pública; v. 39, n 3, p. 467-72, 2005.

DESHPANDE, A.D., BAKER, E.A., LOVEGREEN, S.L., BROWNSON, R.C. Environmental correlates of physical activity among individuals with diabetes in the rural Midwest. Diabetes Care 28, 1012-1018, 2005.

DAWSON J, HILLSDON M, BOLLER I, FOSTER C. Perceived barriers to walking in the neighborhood environment: a survey of middle-aged and older adults. $\mathbf{J}$ Aging Phys Act; 15:318-35, 2007.

FISHER KJ, Li F, MICHAEL Y, CLEVELAND, M. Neighborhood-level influences on physical activity among older adults: a multilevel analysis. J Aging Phys Act, 12:4563,2004

FRUMKIN, H. Healthy places: exploring the evidence. American Journal of Public Health 93 (9), 1451-1456, 2003.

GILES-CORTI B, BROOMHALL MH, KNUIMAN M, et al. Increasing walking: how important is distance to, attractiveness, and size of public open space? American Journal of Prev Medicine; vol. 28(2), Suppl 2, pag.169 -76, 2005.

GODBEY, G.C.; CALDWELL, L.L.; FLOYD, M.; PAYNE, L.L. Contributions of Leisure Studies and Recreation and Park Management Research to the Active Living Agenda. American Journal of Preventive Medicine, vol.28 (2) Suppl. 2, pag.150158, 2005.

GUEDES, I. C. M.; BERTOLI, S. R.,; Zannin, P. H. T. Influence of urban shapes onenvironmental noise: A case study in Aracajú, Brazil. Science of the Total Environment, 412-413, 66-76, 2011.

HANSMANN, R., HUG, S. M., SEELAND, K. Restoration and stress relief through physical activities in forests and parks. Urban Forestry \& Urban Greening 6 (213225), 2007. 
HARNIK, P. The Excellent City Park System: What Makes it Great and How to Get There? San Francisco, published by The Trust Public Land (2003) Reprinted in (2006). Disponível em: www.tpl.org, Acessado em 8 de agosto de 2010.

HERZOG, T.R., CHEN, H.C., PRIMEAU, J.S. Perception of the restorative potential of natural and other settings. Journal of Environmental Psychology 22, 295-306, 2002.

HILDEBRAND, E.; GRAÇA, L.R.; MILANO, M.S.; Distância de Deslocamento dos Visitantes dos Parques Urbanos em Curitiba-Pr. Floresta e Ambiente. Jan./Dez, Vol. 8, n.1, pag.76-83, 2001.

HOEHNER, C.M.; RAMIREZ, L.K. Brennan; ELLIOTT, M.B.; HANDY, S.L.; BROWNSON, R.C. Perceived and Objective Environmental Measures and Physical Activity Among Urban Adults. American Journal of Preventive Medicine; Vol 28, Supply 2, 2005.

HORNIG, E.F., Bringing Family Back to the Park. Parks \& Recreation, Jul. vol 40, suppl. 7; pag. 46, 2005.

KAHN, E.B.; RAMSEY, L.T.; BROWNSON, R.C.; HEATH, G.W.; HOWZE, E.H.; POWELL, K.E.; STONE, E.J. ; RAJAB, M.W.; CORSO, P. and the Task Force on Community Preventive Services. The Effectiveness of Interventions to Increase Physical Activity A Systematic Review. American Journal of Preventive Medicine, vol.22, suppl 4, pag. 73-107, 2002.

KAPLAN, S. The restorative benefits of nature: toward an integrated framework. Journal of Environmental Psychology 15, 169-182, 1995.

KAMPHUIS CB, VAN Lenthe FJ, GISKES K, BRUG J, MACKENBACH JP. Perceived environmental determinants of physical activity and fruit and vegetable consumption among high and low socioeconomic groups in the Netherlands. Health Place; 13:493-503, 2007.

KACZYNSKI, A., HENDERSON, K. Environmental correlates of physical activity: a review of evidence about parks and recreation. Leisure Sciences 29, 315-354, 2007.

KACZYNSKI, A.; HENDERSON, K.A. Parks and Recreation Settings and Active Living: A Review of Associations With Physical Activity Function and Intensity. Journal of Physical Activity and Health, vol.5, pag.619-632, 2008.

KIRCHHOFF, A.C.; ELLIOTT, L.; SCHLICHTING, J.A.; CHIN, M.H. Strategies for physical activity maintenance in african american women. American journal of health behavior. Star City, v. 32, n. 5, p. 517-524, 2008. 
KOHL, H.W. Physical activity and cardiovascular disease: evidence for a dose response. Medicine and Science in Sports and Exercise, 33, S472-83; discussion S493-4, 2001.

LIBRETT, J.; HENDERSON, K.; GODBEY, G.; MORROW, J.R. Jr. An Introduction to Parks, Recreation, and Public Health: Collaborative Frameworks for Promoting Physical Activity. Journal of Physical Activity \& Health, vol. 4, Suppl. 1, pag1-13, 2007.

MILANO, M.S. Avaliação e análise da arborização de ruas de Curitiba-PR, 1984. Dissertação Mestrado-Universidade Federal do Paraná, Curitiba - Paraná.

MASCARÓ, L. J. MASCARÓ, J. Vegetação Urbana. Porto Alegre: UFRGS/FINEP, 242 p, 2002.

MCGINN, A.P.; EVENSON, K.R.; HERRING, A.H.; HUSTON, S.L.; RODRIGUEZ, D.A. Exploring associations between physical activity and perceived and objective measures of the built environment. Journal of urban health: bulletin of the New York Academy of Medicine. Cary, v. 84, n. 2, p.162-84, 2007.

MCCORMACK, G.R.; ROCK, M; TOOHEY, A. M.; HIGNELL, D. Characteristics of urban parks associated with park use and physical activity: A review of qualitative research. Health \& Place 16, 712-726, 2010.

MOWEN, A.; KACZYNSKI, A.; COHEN, D. The Potential of Parks and Recreation in Addressing Physical Activity and Fitness. Research Digest, march, series 9, n 1, 2008.

NUCCI, J.C. Qualidade Ambiental e adensamento urbano. São Paulo: Fapesp, 2001.

NAHAS, M.V. Atividade física, saúde e qualidade de vida: conceitos e sugestões para um estilo de vida ativo. Londrina: Midiograf. 2003.

OWEN, N.; HUMPEL, N.; LESLIE, E.; BAUMAN, A.; SALLIS, J.F. Understanding Environmental Influences on Walking Review and Research Agenda. American Journal of Preventive Medicine, vol. 27 suppl. 1, pag 67-76, 2004.

PRETTY, J., PEACOCK, J., SELLENS, M., GRIFFIN, M. The mental and physical health outcomes of green exercise. International Journal of Environmental Health Research 15 (5), 319-337, 2005.

REIS, R. S. Determinantes Ambientais para a Realização de Atividades Físicas nos Parques Urbanos de Curitiba: Uma Abordagem Sócio-Ecológica da Percepção dos Usuários. Florianópolis, 2001. 101 p. Dissertação (Mestrado em Educação Física) - Centro de Desportos da Universidade Federal de Santa Catarina. 
STAATS, H., KIEVIET, A., HARTIG, T. Where to recover from attentional fatigue: an expectancy value of environmental preference. Journal of Environmental Psychology 23, 147-157, 2003.

SALLIS, J.F.; JOHNSON, M.F.; CALFAS, K.J.; CAPAROSA, S.; NICHOLS, J.F. Assessing Perceived Physical Environmental Variables That May Influence Physical Activity. Research Quarterly for Exercise and Sport; Dec; vol.68, suppl. 4, pag.345, 1997.

SALLIS, J.; CERVERO R.B, ASCHER, W.; HENDERSON, K.A.; M. KRAFT, Katherine; KERR, J. An Ecological Approach to Creating Active Living Communities. Annual Review of Public Health; Vol.27, pag.297-322, 2006.

SHERER, P. The Benefits of Parks: Why America Needs More City Parks and Open Space. The Trust for Public Land, (2003) Disponível em: http://www.tpl.org, Acessado em: 8 de agosto de 2010.

SOUZA, J. M. N. Atividade Física ao Ar Livre e Parques Urbanos. Porto, 2007. 52 p. Monografia em Educação Física - Faculdade de Desporto da Universidade do Porto.

SOARES, H.H.O. A Promoção da Saúde através da Atividade Física em Ambientes Naturais: Um estudo com mulheres adultas residentes na zona leste da cidade de Manaus. Porto, 2009. 109 p. Dissertação de mestrado em atividade física e saúde - Faculdade de Desporto da Universidade do Porto.

STOKOLS, D. Establishing and Maintaining Healthy Environments: Towards a Social Ecology of Health Promotion. American Psychologist, January, 1992.

SZEREMETA, B; ZANNIN, P. H. T. Analysis and evaluation of soundscapes in public parks through interviews and measurement of noise. Science of Total Environment, 497, 6143-6149, 2009.

TESTER, J.; BAKER, R. Making the playfields even: Evaluating the impact of an environmental intervention on park use and physical activity. Preventive Medicine 48, 316-320, 2009.

U.S. Department of Health and Human Services. Physical Activity and Health: A Report of the Surgeon General. Atlanta, GA: U.S. Department of Health and Human Services, Centers for Disease Control and Prevention, National Center for Chronic Disease Prevention and Health Promotion, 1996. Capítulo 3 e 4. Disponível em: http://www.cdc.gov/nccdphp/sgr. Acessado em: 12/07/2010.

WARBURTON, D.E.; NICOL, C.W.; BREDIN, S.S. Health benefits of physical activity: the evidence. Can Med Assoc J., vol. 174, pag. 802-809, 2006.

ZANNIN, P.H.T.; SZEREMETTA, B. Avaliação da poluição sonora no parque Jardim Botânico de Curitiba, Paraná, Brasil. Cadernos de Saúde Pública, v. 19, n 2, p. 683-686, 2003. 
ZANNIN, P.H.T.; SANT'ANA, D.Q. Noise mapping at different stages of a freeway redevelopment project - A case study in Brazil. Applied Acoustics, v. 72 p. 479486, 2011.

ZANNIN, P.H.T.; ENGEL, M.S., FIEDLER, P.E.K., BUNN, F. Characterization of environmental noise based on noise measurements, noise mapping and interviews: A case study at a university campus in Brazil. Cities, v. 31, p. 317-327, 2013. 